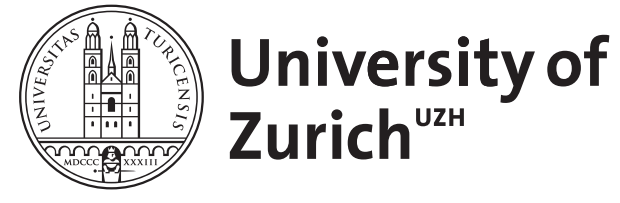

\title{
Ultraschall und Arthritis
}

Tamborrini, G ; Backhaus, M ; Schmidt, W ; Ziswiler, H R

\begin{abstract}
Die Arthrosonographie ist ein etabliertes und validiertes diagnostisches Verfahren in der Rheumatologie. Durch ihren hohen Weichteilkontrast ist die Sonographie in der Lage, Weichteilveränderungen wie z.B. Synovialisveränderungen zu detektieren. Knorpel- oder Knochenveränderungen im Rahmen einer rheumatoiden Arthritis (RA), einer Spondyloarthritis oder einer Kristallarthritis können teilweise nur sonographisch oder in vielen Fällen zu einem früheren Zeitpunkt als mit der konventionellen Bildgebung erfasst werden. Die Aktivität entzündlicher Veränderungen kann mit Hilfe der Doppler- und Power-Dopplersonographie gut dargestellt werden. In der Früharthritisdiagnostik gewinnt die Sonographie zunehmend an Bedeutung, insbesondere bei undifferenzierter Arthritis und bei unauffälligem Röntgenbefund. Neben der Diagnostik der Früharthritis und dem Therapiemonitoring einer RA erlaubt die Sonographie die Darstellung pathognomonischer Veränderungen bei seronegativen Spondyloarthritiden und Kristallablagerungserkrankungen wie Gicht, Chondrokalzinose und Apatitose. Sonographiegesteuerte diagnostische und therapeutische Interventionen zeichnen sich durch eine extrem hohe Treffsicherheit und Verbesserung der klinischen Wirksamkeit verglichen mit ungesteuerten Verfahren aus. Zusammenfassend nimmt die Sonographie zunehmend einen zentralen Stellenwert ein in der Abklärung und Behandlungssteuerung bei entzündlichen Gelenkerkrankungen
\end{abstract}

DOI: https://doi.org/10.1007/s00393-010-0730-1

Other titles: Ultrasound and arthritis

Posted at the Zurich Open Repository and Archive, University of Zurich

ZORA URL: https://doi.org/10.5167/uzh-156174

Journal Article

Published Version

Originally published at:

Tamborrini, G; Backhaus, M; Schmidt, W; Ziswiler, H R (2010). Ultraschall und Arthritis. Zeitschrift für Rheumatologie, 69(10):889-902.

DOI: https://doi.org/10.1007/s00393-010-0730-1 
Z Rheumatol 2010 · 69:889-902

DOI 10.1007/s00393-010-0730-1

c) Springer-Verlag 2010

\section{Redaktion}

J. Wollenhaupt, Hamburg (Leitung)

O. Distler, Zürich

J. Grifka, Bad Abbach

G. Schett, Erlangen

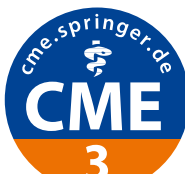

\section{Punkten Sie online auf}

\section{CME.springer.de}

\section{Teilnahmemöglichkeiten}

- kostenfrei im Rahmen des jeweiligen Zeitschriftenabonnements

- individuelle Teilnahme durch den Erwerb von CME.Tickets auf CME.springer.de

\section{Zertifizierung}

Diese Fortbildungseinheit ist mit 3 CME-Punkten zertifiziert von der Landesärztekammer Hessen und der Nordrheinischen Akademie für Ärztliche Fort- und Weiterbildung und damit auch für andere Ärztekammern anerkennungsfähig.

\section{Hinweis für Leser aus Österreich} Gemäß dem Diplom-Fortbildungs-Programm (DFP) der Österreichischen Ärztekammer werden die auf CME.springer.de erworbenen CME-Punkte hierfür 1:1 als fachspezifische Fortbildung anerkannt.

\section{Kontakt und weitere Informationen}

Springer-Verlag GmbH

Fachzeitschriften Medizin / Psychologie

CME-Helpdesk, Tiergartenstraße 17

69121 Heidelberg

E-Mail: cme@springer.com

CME.springer.de
G. Tamborrini' $\cdot$ M. Backhaus ${ }^{2} \cdot$ W. Schmidt ${ }^{3} \cdot$ H.R. Ziswiler ${ }^{4}$

${ }^{1}$ Rheumaklinik, UniversitätsSpital Zürich

${ }^{2}$ Medizinische Klinik mit Schwerpunkt Rheumatologie und Klinische Immunologie, Charité-Universitätsmedizin Berlin

${ }^{3}$ Rheumaklinik Berlin-Buch, Immanuel Krankenhaus GmbH, Berlin-Buch

${ }^{4}$ Klinik für Rheumatologie, klinische Immunologie, Allergologie, Universitätsspital Insel, Bern

\section{Ultraschall und Arthritis}

\section{Zusammenfassung}

Die Arthrosonographie ist ein etabliertes und validiertes diagnostisches Verfahren in der Rheumatologie. Durch ihren hohen Weichteilkontrast ist die Sonographie in der Lage, Weichteilveränderungen wie z. B. Synovialisveränderungen zu detektieren. Knorpel- oder Knochenveränderungen im Rahmen einer rheumatoiden Arthritis (RA), einer Spondyloarthritis oder einer Kristallarthritis können teilweise nur sonographisch oder in vielen Fällen zu einem früheren Zeitpunkt als mit der konventionellen Bildgebung erfasst werden. Die Aktivität entzündlicher Veränderungen kann mit Hilfe der Doppler- und Power-Dopplersonographie gut dargestellt werden. In der Früharthritisdiagnostik gewinnt die Sonographie zunehmend an Bedeutung, insbesondere bei undifferenzierter Arthritis und bei unauffälligem Röntgenbefund. Neben der Diagnostik der Früharthritis und dem Therapiemonitoring einer RA erlaubt die Sonographie die Darstellung pathognomonischer Veränderungen bei seronegativen Spondyloarthritiden und Kristallablagerungserkrankungen wie Gicht, Chondrokalzinose und Apatitose. Sonographiegesteuerte diagnostische und therapeutische Interventionen zeichnen sich durch eine extrem hohe Treffsicherheit und Verbesserung der klinischen Wirksamkeit verglichen mit ungesteuerten Verfahren aus. Zusammenfassend nimmt die Sonographie zunehmend einen zentralen Stellenwert ein in der Abklärung und Behandlungssteuerung bei entzündlichen Gelenkerkrankungen.

\section{Schlüsselwörter}

Ultraschall · Rheumatide Arthritis · Spondyloarthritis · Kristallarthropathien · Injektion

\section{Ultrasound and arthritis}

\begin{abstract}
Musculoskeletal ultrasonography (US) is an established and validated imaging technique in rheumatology. Ultrasonography is able to directly visualize soft tissue pathologies such as synovial tissue changes. Pathological findings in superficial cartilage, bone lesions and synovial tissue changes in the context of rheumatoid arthritis, spondyloarthritis or crystal arthropathies may only be seen by sonography or detected earlier by ultrasonography compared to conventional imaging techniques. The activity of an inflammatory arthropathy can be visualized using Doppler and power Doppler US. US is helpful in the detection of early inflammatory changes, particularly in patients with undifferentiated arthritis and/or unremarkable conventional radiography. In addition to diagnosis in early arthritis and monitoring of therapy in rheumatoid arthritis, sonography is able to detect pivotal pathologies in spondyloarthritis and crystal deposition diseases such as gout, pseudogout and apatite deposition disease. Ultrasound-guided diagnostic and therapeutic interventions are characterized by their excellent accuracy and improvement of clinical effectiveness compared to unguided procedures. In conclusion, ultrasonography plays a pivotal role in the assessment and monitoring of therapy in rheumatic diseases.
\end{abstract}

\section{Keywords}

Ultrasound $\cdot$ Rheumatoid arthritis $\cdot$ Spondyloarthritis $\cdot$ Crystal arthropathy $\cdot$ Injection

Die Autoren des vorliegenden Beitrags waren an der Manuskripterstellung folgendermaßen beteiligt: Rheumatoide Arthritis: M. Backhaus; Spondyloarthritis: H.R. Ziswiler; Kristallarthropathien: W. Schmidt; Interventionen: G Tamborrini 
Der Ultraschall kann sowohl den entzündlichen Weichteil- als auch den entzündlichen Knochenprozess bei arthritischen Erkrankungen erfassen

\section{- Früharthritisdiagnostik}

Die Sonographie gestattet eine Differenzierung exsudativer und proliferativer Synovialisveränderungen

\section{- Tenosynovitis}

Eine Synovialisproliferation geht häufig mit einer entzündlichen Ergussbildung einher
Die Sonographie des Bewegungsapparates erbringt in unmittelbarer Ergänzung zur klinischen Untersuchung zusätzliche diagnostische Informationen und erleichtert therapeutische Maßnahmen. Die Arthrosonographie, als unbelastende und vergleichsweise kostengünstige Untersuchung, bietet sich insbesondere für die bildgebende Diagnostik der Weichteile oder der Gelenke an. Die Arthrosonographie ist nicht nur in der Arthritisdiagnostik ein anerkanntes und validiertes Werkzeug, sondern eignet sich auch zum Monitoring der Therapie (z. B. Abnahme von Synovitiden bei der rheumatoiden Arthritis) und für Interventionen (diagnostische Punktionen und therapeutische Infiltrationen). Die heutigen hochauflösenden Ultraschallsonden erlauben eine zuverlässige Beurteilung kleiner Gelenke, des Knorpels oder der Enthesen. Anhand der Lektüre dieses Beitrags erfährt der Leser den aktuellen Stellenwert der Sonographie bei der rheumatoiden Arthritis (RA), den Kristallarthropathien, den Spondyloarthritiden und bei Interventionen.

\section{Rheumatoide Arthritis}

Die RA ist eine chronisch entzündliche Gelenkerkrankung, die unbehandelt sehr rasch zur Gelenkzerstörung führen kann. Der Krankheitsverlauf wird vom Zeitpunkt der Diagnosestellung und dem Therapiebeginn mit hochwirksamen krankheitsmodifizierenden antirheumatischen Medikamenten („disease modifying antirheumatic drugs“, DMARDs) bestimmt. Die bildgebende Säule ist neben der Erfassung der klinischen Daten und der Laborparameter entscheidend für die Erfassung der strukturellen Gelenkveränderungen. Die konventionelle Röntgendiagnostik galt bisher als der Referenzstandard für den Nachweis des destruierenden Gelenkprozesses. Strukturelle Gelenkveränderungen zeigen sich jedoch erst etwa 6 Monate nach Krankheitsbeginn im konventionellen Röntgenbild. Der Ultraschall ist in der Lage, frühzeitiger sowohl den entzündlichen Weichteil- als auch den entzündlichen Knochenprozess bei arthritischen Erkrankungen zu erfassen [2]. Die Synovitis nimmt einen zentralen Platz im gelenkzerstörenden Prozess ein. Studien haben gezeigt, dass keine Knochenschädigung ohne Synovitis auftritt. Die frühe Detektion der synovialen Proliferation ist im Rahmen der - Früharthritisdiagnostik von besonderer Bedeutung, die wiederum der Ultraschall ermöglicht.

Die sonographischen Befunde nehmen Einfluss auf das weitere diagnostische und therapeutische Vorgehen [19]. Die Sonographie gestattet infolge ihres guten Weichteilkontrasts eine Differenzierung exsudativer und proliferativer Synovialisveränderungen sowie von Sehnenscheidenentzündungen. Oberflächlich liegende Knorpel-Knochen-Läsionen/Erosionen können sonographisch frühzeitiger als mittels konventioneller Röntgendiagnostik beispielsweise am Schulter-, Hand- und Fußskelett dargestellt werden $[3,44]$.

\section{Sonographische Differenzierung der Weichteil- und Knochenläsionen}

Die Zeichen für entzündliche Weichteile umfassen die Synovitis mit oder ohne Erguss und mit synovialer Hypertrophie als Zeichen einer entzündlichen Infiltration der Synovia sowie die $>$ Tenosynovitis mit Erguss und synovialer Verdickung im Bereich der Sehnenscheiden. Pathophysiologisch geht eine Synovialisproliferation häufig mit einer entzündlichen Ergussbildung einher, so dass beide Phänomene parallel auftreten können und die Kombination aus beiden Pathologika als Synovitis bezeichnet wird. Die entzündlichen Knochenläsionen/-erosionen präsentieren sich als Konturunregelmäßigkeit oder in Form von Konturunterbrechungen der Gelenkoberfläche in zwei zueinander senkrecht stehenden Ebenen in oberflächlichen Regionen, die der Arthrosonographie zugänglich sind.

Auf der 7. OMERACT- („Outcome Measurement Rheumatoid Arthritis Clinical Trial“-) Konferenz im Mai 2004 in Monterrey, Kalifornien, wurden die arthrosonographisch erfassbaren Befunde für die RA wie folgt definiert [43]:

\section{Erguss}

Sonographisch stellt sich ein Erguss als echoarmes oder echofreies intraartikuläres Material dar, welches verschiebbar und komprimierbar ist und keine Dopplersignale aufweist. Das Echomuster verhält sich immer relativ zum subdermalen Fettgewebe. 

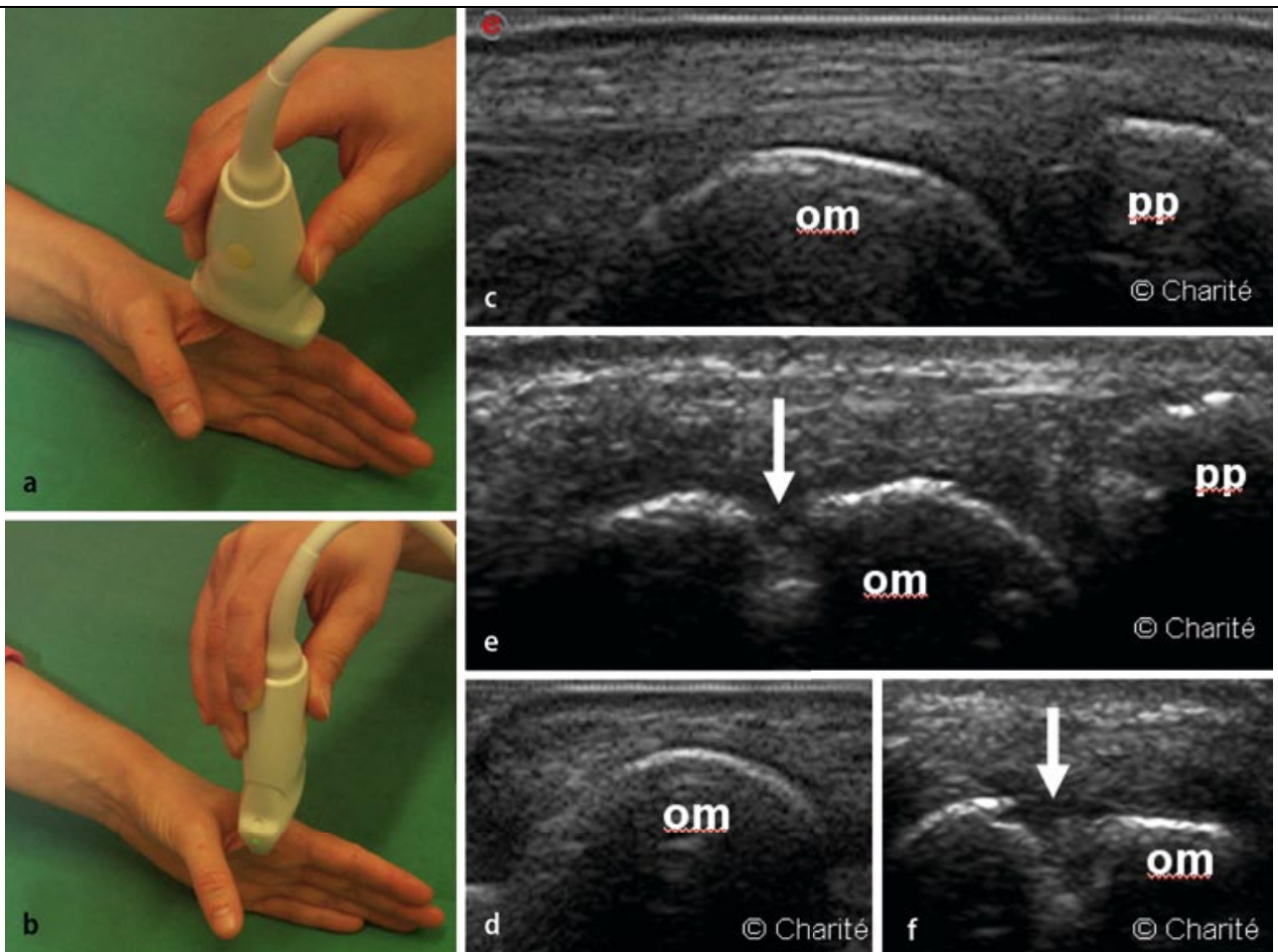

Abb. $1 \Delta$ Erosion. Schallkopfposition a im Longitudinalschnitt und b im Transversalschnitt. c, d US-Normalbefund. e, $\mathbf{f}$ Erosion am Os-metacarpale-MCP-Gelenk II radial (om Caput Os metacarpale, $p p$ Basis Phalanx proximalis)

\section{Synoviale Hypertrophie/Synovialisproliferation}

Hier handelt es sich um abnormales echoarmes intraartikuläres Gewebe, das sich nicht verschieben lässt, kaum komprimierbar ist und Dopplersignale aufweisen kann. Das Echomuster verhält sich immer relativ zum subdermalen Fettgewebe und kann gelegentlich auch echogen oder echoreich sein.

\section{Tenosynovitis}

Eine Tenosynovitis ist charakterisiert durch echoarmes oder echofreies verdicktes Gewebe/Material mit oder ohne Flüssigkeit innerhalb der Sehnenscheide mit möglichen Dopplersignalen, darstellbar in zwei zueinander senkrecht stehenden Ebenen.

\section{Erosion}

Bei der Erosion handelt es sich um eine intraartikuläre Diskontinuität der Knochenoberfläche, die in zwei zueinander senkrecht stehenden Ebenen darstellbar ist (• Abb. 1 a-f)

\section{Ultraschall-Assessment bei rheumatoider Arthritis}

Klinische Studien haben gezeigt, dass der Ultraschall sensitiver im Aufdecken von Entzündungszeichen ist als die klinische Untersuchung $[3,2,30]$ und somit eine höhere $>$ diagnostische Wertigkeit hat. Von 60 untersuchten Patienten mit arthritischen Fingerbeschwerden zeigten über 20\% der untersuchten Fingergelenke $(\mathrm{n}=840)$ bereits sonographisch Synovitiszeichen, obwohl sie bezüglich Druckschmerz und Gelenkschwellung klinisch noch stumm waren [3]. Synovitiszeichen sind im Bereich der Fingergelenke häufiger palmar proximal nachweisbar als dorsalseitig [29]. — Synovitis-Scores wurden zur Erfassung des arthritischen Prozesses am Handskelett bereits entwickelt [29, 35]. Während die Arbeitsgruppe von Szkudlarek [35] die entzündlichen Weichteilveränderungen getrennt nach Erguss und synovialer Hypertrophie graduiert, fasst die Arbeitsgruppe von Scheel [29, 31] den Erguss und die synoviale Hypertrophie in einem Score zusammen. Es konnte gezeigt werden, dass es statistisch keinen Unterschied gab zwischen dem semiquantitativen Score aus 6 und 8 von palmar untersuchten Fingergelenken [Metakarpophalangeal- (MCP-) und proximales Interphalangeal- (PIP-) Gelenk II-IV oder II-V] zur Erfassung des Arthritisprozesses [29]. Ebenfalls konnte gezeigt werden, dass der semiquantitative Synovitis-Score [Grad 0: normal, Grad 1: minimale Synovitis (Erguss/Hy-
Der Ultraschall ist sensitiver im Aufdecken von Entzündungszeichen als die klinische Untersuchung

\section{Diagnostische Wertigkeit}

\section{- Synovitis-Scores}


Entzündliche Weichteilprozesse

- Processus styloideus

Erosive Läsionen an den Fingergelenken lassen sich besonders gut radialseitig an den MCP-Gelenken I und II und ulnarseitig am MCP-Gelenk V erfassen

\section{- Zehengrundgelenke}

\section{- Poplitealzyste}

\section{- Entzündungsaktivität}

Die Power-Dopplersonographie ist für Verlaufskontrollen von BiologikaTherapien sehr gut geeignet

\section{- Aktivitätsbeurteilung}

Die Arthrosonographie ist hilfreich in der Früharthritisdiagnostik, insbesondere bei Patienten mit unauffälligem Röntgenbefund und suspekter Klinik

\section{Enthesitis}

pertrophie), Grad 2: mäßige Synovitis, Grad 3: starke Synovitis] gut mit dem quantitativen SynovitisScore korreliert. Der Grenzwert zwischen „gesund“ und „pathologisch“ liegt für MCP- und PIP-Gelenke nach ROC- („receiver operating characteristics“-) Analysen bei 0,6 mm, für den Grad 1/2 bei $1,75 \mathrm{~mm}$ bzw. $1,15 \mathrm{~mm}$ und für den Grad 2/3 bei $\geq 2,08 \mathrm{~mm}$ bzw. $\geq 1,83 \mathrm{~mm}$ [29].

Der entzündliche Weichteilprozess lässt sich auch im Handgelenk sehr frühzeitig allseitig erfassen. Insbesondere im Bereich des $>$ Processus styloideus und entlang der Sehne des M. extensor carpi ulnaris zeigen sich sonographisch frühzeitig Entzündungszeichen bei Patienten mit RA [4].

Erosive Knochenläsionen lassen sich sowohl im Bereich der Handwurzelknochen als auch an den Fingergelenken frühzeitiger darstellen als im konventionellen Röntgenbild. Während die genaue anatomische Zuordnung der Erosionen an den Handwurzelknochen sich sonographisch etwas schwierig gestaltet, lassen sich erosive Läsionen an den Fingergelenken besonders gut radialseitig an den MCP-Gelenken I und II und ulnarseitig am MCP-Gelenk V erfassen [44]. Aber auch dorsalseitig insbesondere bei Flexionsstellung in Höhe der MCP- und PIP-Gelenke lassen sich erosive Läsionen frühzeitiger erfassen als mittels konventioneller Röntgendiagnostik [3, 2, 30]. Mit hochauflösenden Schallsonden konnten im Bereich der PIP-Gelenke bei RA-Patienten mittels Arthrosonographie mehr erosive Knochenläsionen nachgewiesen werden als mittels 0,2 Tesla (T) Magnetresonanztomographie (MRT; [30, 37]).

Der synovitische Prozess kann ebenfalls an den Füßen insbesondere an den Zehengrundgelenken [Metatarsophalangeal- (MTP-)Gelenke] gut erfasst werden [36], aber prinzipiell auch in jedem anderen peripheren Gelenk. Das ist ein erheblicher Vorteil der Arthrosonographie. Bei der Abklärung eines Wadenkompressionssyndroms ist die sonographische Erfassung einer $>$ Poplitealzyste in der Kniekehle sehr hilfreich. Diese kann wiederum durch Kompression auf das Gefäßband sekundär für die Entwicklung einer Thrombose verantwortlich sein.

Mit Hilfe der Doppler- und Power-Dopplersonographie ist es möglich, die $>$ Entzündungsaktivität arthritischer Prozesse zu beurteilen. Hierbei sind die intraartikulären bzw. intrasynovialen Farbsignale von entscheidender Bedeutung. Davon zu unterscheiden sind die periartikulären Farbsignale infolge einer Hyperämie bei arthritischen Prozessen.

Zum Einsatz der Doppler- und Power-Dopplersonographie gibt es verschiedene Studien bei Patienten mit RA an den Händen und Kniegelenken, die eine gute Korrelation im Vergleich zur Klinik, zur MRT mit Kontrastmittel sowie zur Histopathologie zeigten [28, 38, 45]. Die Power-Dopplersonographie ist auch für Therapieverlaufskontrollen mit neuen Therapieverfahren (Biologika) sehr gut geeignet.

Für die Aktivitätsbeurteilung setzt sich zunehmend ein vierstufiges semiquantitatives Grading der Farbpixel durch [34].

- Grad o: kein erkennbares Dopplersignal (Farbpixel)/kein Fluss,

- Grad 1: einzelne Dopplersignale/wenig Fluss,

- Grad 2: mehrere Dopplersignale/deutlicher Fluss,

- Grad 3: nahezu komplette Ausfüllung des Gelenkbereichs mit konfluierenden Dopplersignalen/ starker Fluss.

\section{Schlussfolgerung}

Die Arthrosonographie ist hilfreich in der Früharthritisdiagnostik insbesondere bei Patienten mit unauffälligem Röntgenbefund und suspekter Klinik. Sie ermöglicht eine frühzeitige Erfassung des synovitischen Prozesses, erlaubt eine rasche Verlaufsbeurteilung und damit eine gute Therapieverlaufskontrolle. Durch den Einsatz der Doppler- und Power-Dopplersonographie können zusätzliche Informationen zur Aktivität des entzündlichen Gelenkprozesses erzielt werden. Es ist ein patientenfreundliches Verfahren mit hohem Aussagewert und ist fester Bestandteil in der Abklärung arthritischer Krankheitsbilder.

\section{Spondyloarthritis}

Die heterogene Erkrankungsgruppe der mit dem HLA-B27-Antigen assoziierten Spondyloarthritiden (SpA) umfasst den axialen Typ der SpA (ankylosierende Spondylitis, AS), den peripheren Typ, die psoriatische Arthritis (PsA), reaktive Arthritis, enteropathische Arthritis und die undifferenzierte SpA. Pathomorphologisch charakteristisches Merkmal der SpA ist die Enthesitis, die Entzün- 


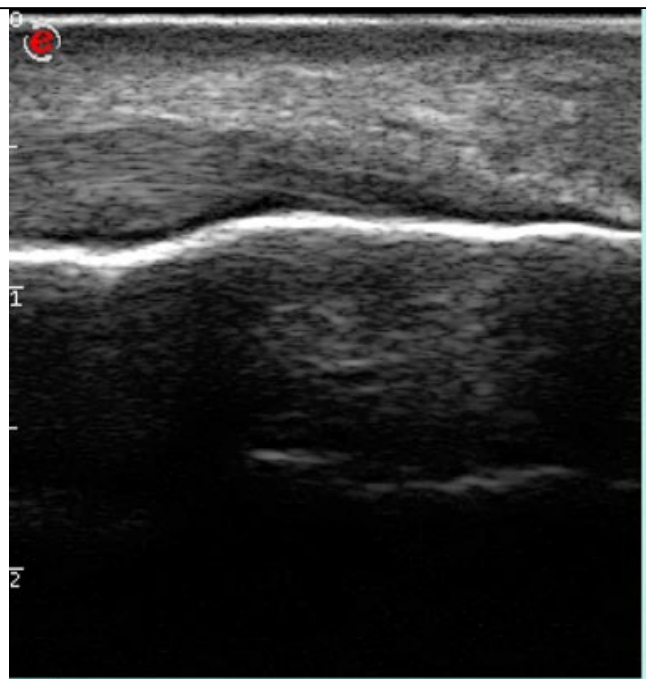

Links

Abb. $2 \Delta$ Rechte Achillessehne im Graustufenbild ödematös verdickt, hyperechogene streifenförmige Echotexturstörung, Kortikalis intakt

dung an der Ansatzstelle von Sehnen, Bändern und Gelenkskapseln in den Knochen. Synovitiden und Osteitiden sind weitere charakteristische Merkmale der SpA, diese sind aber meistens in unmittelbarer Nachbarschaft zu der Enthese mit Enthesitis [22].

Periphere Enthesitiden, z. B. an der Achillessehne, sind meistens klinisch diagnostizierbar. Enthesen im Bereich kleiner Gelenke, in der Tiefe des Gewebes gelegene Enthesen großer Gelenke und jene an der Wirbelsäule sind klinisch jedoch nicht beurteilbar. Sehr frühe und diskrete entzündliche Veränderungen, welche mit oder ohne klinische Symptome ablaufen können, sind oft weder klinisch noch mit konventioneller Röntgenbildtechnik erkennbar. Mit der Etablierung moderner, sehr wirksamer Therapiemodalitäten, wie den Tumor-Nekrose-Faktor- (TNF-)a-Hemmern, wird die Notwendigkeit einer adäquaten frühzeitigen Diagnostik und eines adäquaten Therapiemonitorings immer dringender. Sowohl mittels MRT als auch mit Ultraschall sind die pathognomonischen Weichteilveränderungen der Enthesen frühzeitig und spezifisch erfassbar.

\section{Enthesitis}

Das Konzept des „Enthese-Organs“, bestehend aus dem Knochen an der Enthese, einer faserknorpeligen Übergangszone und allen ansetzenden Weichteilen, hat sich weitgehend durchgesetzt. Die Enthese kann aufgeteilt werden in die eigentliche Weichteilinsertionszone, benachbartes Periost mit Faserknorpel und den darunterliegenden Knochen [24]. Einige Strukturen sind zwar nicht typische Enthesen im engeren Sinn, sie verhalten sich jedoch aufgrund vergleichbarer anatomischer, histologischer und biomechanischer Eigenheiten wie Enthesen. Solche „funktionellen Enthesen“ sind die Sakroiliakal- und Akromioklavikulargelenke sowie vollständig eingehüllte Sehnen wie jene in der Perimalleolarregion der Sprunggelenke.

Typische Veränderungen an der Enthese im Ultraschall-B-Bild sind Ödem und Verdickung, intratendinöse fokale Veränderungen mit hypo- oder hyperechogenen Zonen, Kalzifikationen, periostal-knöcherne Ausziehungen (Enthesophyten) oder Defekte (Erosionen) und Bursitiden. Enthesitiden haben eine Prädilektion für die unteren Extremitäten, kommen jedoch auch an oberen Extremitäten vor. Am häufigsten ist die Verdickung/ödematöse Auftreibung erkennbar (• Abb. 2). Schon in sehr frühen Untersuchungen waren 50\% der Enthesen, die sonographisch nur diese Veränderung aufwiesen, klinisch asymptomatisch [21].

Im „Glasgow Ultraschall Enthesitis Score“ (GUESS) werden Sehnenansätze am Patellaoberpol und -unterpol, an der Tuberositas tibiae und am kalkanearen Ansatz von Achillessehne und Plantarfaszie (5 Enthesen) jeweils die Kriterien der Sehnendicke, Bursitis, Knochenerosion und Entesophyt beurteilt. Der GUESS und auch andere vorgeschlagene B-Bild-Ultraschall-Scores sind nicht nur viel sensitiver, sondern auch viel spezifischer als die klinische Untersuchung und dabei gut reproduzier-

\section{- Osteitis}

Enthesen im Bereich kleiner Gelenke sind klinisch nicht beurteilbar

Das „Enthese-Organ“ besteht aus dem Knochen an der Enthese, einer faserknorpeligen Übergangszone und allen ansetzenden Weichteilen

Enthesitiden haben eine Prädilektion für die unteren Extremitäten

\section{„Glasgow Ultraschall Enthesitis} Score" 
Typisch für seronegative $\mathrm{SpA}$ ist die Mehrdurchblutung in der Insertionszone der Sehne

\section{Die Daktylitis ist häufig mit SpA assoziiert}

Die Power-Dopplersonographie eignet sich zum Therapiemonitoring bei Patienten mit Psoriasisarthritis

Enthesophyten am Achillessehnenansatz entstehen eher distal, Erosionen eher proximal

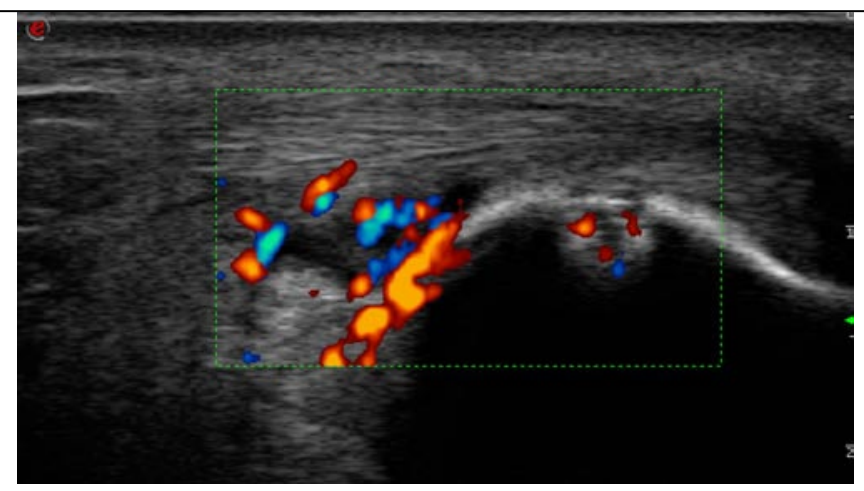

Abb. $3<$ Power-Dopplermodus rechte Achillessehne (gleicher Patient, gleicher Zeitpunkt wie Abb. 2): deutliche Mehrvaskularisation in der verdickten Bursa praeachillea und innerhalb der ossären Läsion bar [6]. Ein großer Vorteil des GUESS ist dessen Einfachheit, er ist daher auch im klinischen Alltag sehr gut anwendbar.

Bei der Untersuchung multipler Enthesen finden sich in 98\% aller SpA-Patienten pathologische Befunde. Im Vergleich dazu hatten nur die Hälfte der Kontrollen (52\%) pathologische Befunde im BBild. Ergänzend zur B-Bild-Morphologie zeigt die Power-Dopplerdarstellung eine Perfusionssteigerung und Neovaskularisation an der Enthese. Erhöhter Blutfluss, dessen Lokalisation und eine quantitative oder semiquantitative Graduierung helfen in der Unterscheidung primär entzündlicher Pathologien bei SpA und rein degenerativen Sehnenaffektionen und sind vielversprechende Verlaufsparameter für Therapiestudien. Typisch für seronegative SpA ist die Mehrdurchblutung in der Insertionszone der Sehne in den Knochen oder in der benachbarten Bursa ( $\bullet$ Abb. 3). Dies steht im Gegensatz zu degenerativen Sehnenproblemen, bei welchen die Mehrdurchblutung praktisch nie an der Insertion, sondern vorwiegend im Sehnenkörper liegt und viel seltener erkennbar ist $[10,9,13]$.

\section{Daktylitis}

Die Daktylitis beschreibt den klinischen Befund einer diffusen Finger- oder Zehenschwellung. Dieser Befund ist häufig aber nicht alleinig assoziiert mit SpA. Der Daktylitis können eine Tenosynovitis, peritendinöses Ödem, Gelenksynovitiden, Enthesitiden, juxtaartikuläre periostale Reaktionen und positive Dopplersignale der Fingerkuppen zugrunde liegen [17]. In unserer Erfahrung liegt am häufigsten eine Tenosynovitis vor.

\section{Synovitis}

Die OMERACT-Kriterien zur Beurteilung synovialer Pathologien kommen wie bei anderen Arthritiden zur Anwendung. Das B-Bild detektiert Erguss und synoviale Proliferationen problemlos auch an distalen Interphalangeal- (DIP-)Gelenken, welche vor allem bei Psoriasisarthritis häufig beteiligt sind und daher mitbeurteilt werden müssen. Die Power-Dopplersonographie hilft bei der Identifikation entzündlicher Aktivität und eignet sich zum Therapiemonitoring bei Patienten mit Psoriasisarthritis [16]

\section{Erosion und Enthesophyt}

Die Erosion wird ebenfalls gemäß OMERACT-Kriterien definiert als Unterbrechung der Knochenoberfläche, darstellbar in zwei senkrecht zueinander stehenden Ebenen ( $\bullet$ Abb. 4 a, b). Der entzündliche Knochensporn (Enthesophyt) ist ebenfalls sehr gut darstellbar. Er steht über dem Niveau der kortikalen Knochenoberfläche und bricht abrupt ab (Sprungschanzenphänomen). Auch diese Pathologie soll in zwei senkrecht zueinander stehenden Ebenen darstellbar sein. Mit Ultraschall konnte gezeigt werden, dass Enthesophyten, bzw. Knochenneubildung, am Achillessehnenansatz eher distal, Erosionen eher proximal entstehen. Diese topographische Verteilung ist möglicherweise pathophysiologisch mit höherer Zugeinwirkung distal am Sehnenansatz erklärbar [23]. Oft liegen Erosionen und osteoproliferative Veränderungen unmittelbar nebeneinander, was z. B. bei Psoriasisarthritis mit Befall von Fingergelenken zu eindrücklichen Befunden im B-Bild führen kann. 
Abb. $4 \triangleright$ Graustufenbild rechte Achillessehne (gleicher Patient wie Abb. 2, 3 Monate später): Bursits praeachillea, zwei benachbarte Erosionen am Achillessehnenansatz, a längs b quer
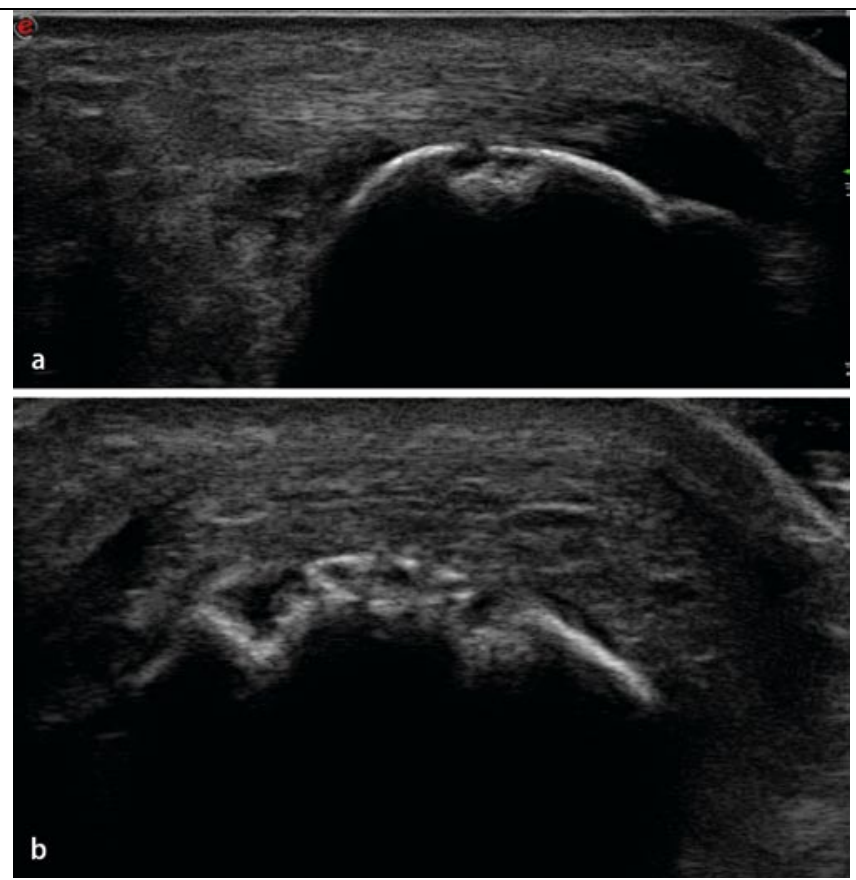

\section{Sakroiliakalgelenk und Spondylitis}

Mit Farb- und Duplexdoppler-Ultraschall kann eine Entzündung am Sakroiliakalgelenk (SIG) bzw. die Enthesitis an den kräftigen sakroilikalen Bandstrukturen nachgewiesen werden. Arslan benutzt dazu den Wiederstandsindex (RI) einer Arterie neben der hinteren Portion der SIG: Ein verminderter RI ist dabei ein indirekter Hinweis für vorhandene Entzündung [1]. Die Messung des RI an den SIG und zusätzlich in paraspinalen Arealen lumbal und thorakal korrespondiert mit der Krankheitsaktivität bei SpA gemessen mit dem BASDAI („Bath Ankylosing Sondylitis Disease Activity Index“) und bessert sich parallel zu den klinischen Parametern unter TNF- $\alpha$-Hemmer-Therapie [41]. Mittels kontrastmittelverstärktem > Farbdoppler-Ultraschall („Contrast Enhanced Ultrasound“, CEUS) wird, gemessen am Goldstandard einer kontrastverstärkten MRT, eine Sensitivität von $94 \%$ und eine Spezifität von $86 \%$ erreicht. Die hohe negative Vorhersagekraft (NPV) von 97\% suggeriert ein gutes Screening-Instrument [20]. Diese Aussicht wird jedoch etwas getrübt durch die niedrige Spezifität und die Tatsache, dass Kontrastmittel gegeben werden muss und im Gegensatz zu einer MRT ein Großteil der gesamten SIG nicht abgebildet werden kann. Zusammenfassend kann der Ultraschall an SIG und der Wirbelsäule im Gegensatz zu peripheren Lokalisationen mit der MRT nicht konkurrieren.

\section{Schlussfolgerung}

Das Potenzial des hochauflösenden Ultraschalls zur Erfassung und Verlaufsbeurteilung von Enthesitis, Synovitis, Daktylitis, Erosionen und Enthesophyten an verschiedenen Lokalisationen unterstreicht die mögliche Rolle, die der Ultraschall für die Diagnose und das Therapiemonitoring bei SpA einnehmen kann. Im klinischen Alltag wird dieses Potenzial vielerorts bereits gezielt genutzt.

Die Erstellung eines umfassenden Ultraschall-SpA-Scores, welcher den OMERACT-Kriterien genügt, die Basispathologien erfasst und die wichtigsten anatomischen Lokalisation mit vernünftigem Zeitaufwand abzubilden erlaubt, ist eine der wichtigen Herausforderungen auf der Forschungsagenda.

\section{Kristallarthropathien}

Die Sonographie hat eine wesentliche Bedeutung in der Diagnostik der Kristallarthropathien. Dabei handelt es sich vor allem um die $\triangleright$ Gicht und die $>$ Pseudogicht (Chondrokalzinose). Außerdem lassen sich sonographisch hervorragend Hydroxyapatitkristallablagerungen z. B. in der Rotatorenman-

Ein verminderter Rl ist ein indirekter Hinweis für eine vorhandene Entzündung

\section{- Farbdoppler-Ultraschall}

Der Ultraschall an SIG und der Wirbelsäule kann mit der MRT nicht konkurrieren

\footnotetext{
Gicht

$\checkmark$ Pseudogicht
} 
Die Sonographie ist insbesondere zum Nachweis einer Gicht wesentlich sensitiver als die Röntgenuntersuchung

Die intraartikuläre Ablagerung von Uratkristallen ist assoziiert mit der Entwicklung von Erosionen

\section{Gichtarthritis}

Die Synovitis bei der Gicht ist typischerweise echoreicher als das umgebende Bindegewebe oder mindestens echogleich

\section{- Gichttophi}

\section{- Polarisationsmikroskop}

Akute Gicht und eitrige Arthritis oder Weichteilinfektionen sind klinisch oft sehr ähnlich

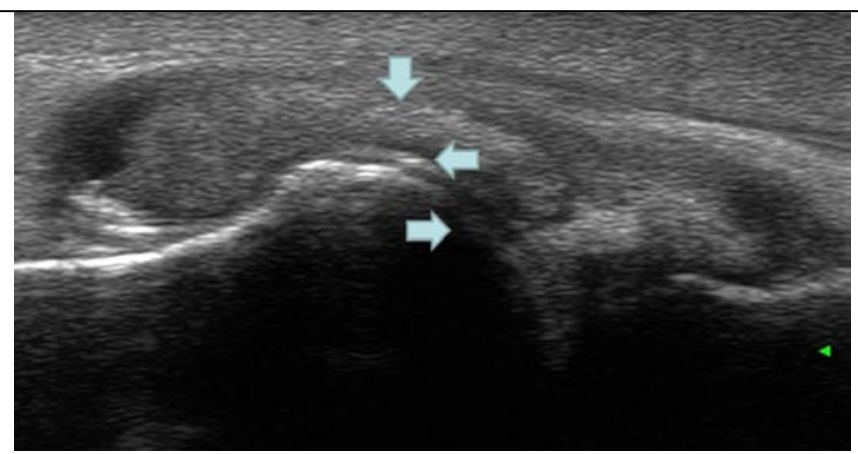

Abb. $5<$ Dorsaler Längsschnitt eines Großzehengrundgelenks bei Gicht. Die verdickte Synovialis ist echoreich $(\downarrow)$ mit dorsaler Schallauslöschung $(\rightarrow)$ und Doppelkonturzeichen $(\leftarrow)$

schette oder im Ansatzbereich der Sehne des M. gluteus medius und minimus am Trochanter major nachweisen. Bis vor wenigen Jahren bestand die Auffassung, dass die konventionelle Röntgendiagnostik die bildgebende Methode der Wahl in der Diagnostik der Kristallarthropathien sei. Inzwischen konnte jedoch gezeigt werden, dass die Sonographie insbesondere zum Nachweis einer Gicht wesentlich sensitiver als die Röntgenuntersuchung ist [11, 27, 46]. Bei guten Kenntnissen in der Sonographie, Verwendung moderner Technik und gründlicher Untersuchung lassen sich auch Chondrokalzinose und Hydroxylapatitablagerungen besser sonographisch als radiologisch nachweisen.

Im Folgenden wird auf die Bedeutung der Sonographie in der Diagnostik der Gicht und der Chondrokalzinose eingegangen. Die intraartikuläre Ablagerung von Uratkristallen ist assoziiert mit der Entwicklung von Erosionen. Die Gichtarthritis ist insbesondere mit der Aktivierung von Interleukin 1 assoziiert. Tophöses Gewebe erodiert den darunter liegenden Knochen [32].

Im Falle einer Gichtarthritis finden sich folgende typische sonographische Befunde (- Abb. 5):

- wolkige echoreiche intraartikuläre Strukturen,

- diese bewirken eine teilweise oder komplette dorsale Schallauslöschung, sodass der darunter liegende Knochen geringer oder nicht sichtbar ist,

- echoreiches Band im Übergangsbereich zwischen Knorpel und Synovium („Doppelkonturzeichen“); das Band zeigt dabei eine ähnliche Echogenität und Dicke wie der darunter liegende Knochen; normalerweise ist diese Linie sehr schmal und entspricht Kristallablagerungen auf dem hyalinen Knorpel,

- intraartikuläre Hyperperfusion bei aktiver Synovitis,

- Erosion bei chronischer oder rezidivierender Synovitis.

Die ersten drei Befunde sind charakteristisch für eine Gicht und erlauben im Zusammenhang mit dem klinischen Befund die Diagnosestellung [39]. Die wissenschaftliche Datenlage ist jedoch noch nicht ausreichend, um zu empfehlen, dass bei positivem Sonographiebefund grundsätzlich auf die Synovialisanalyse unter dem Polarisationsmikroskop verzichtet werden kann. Insgesamt ist die Synovitis bei der Gicht typischerweise echoreicher als das umgebende Bindegewebe oder mindestens echogleich, während die Synovitis bei anderen rheumatischen Erkrankungen wie bei der RA deutlich echoärmer als das umgebende Gewebe ist.

- Gichttophi sind subkutan lokalisiert. Manchmal kommen sie genauso wie Rheumaknoten an den Streckseiten der Unterarme vor. Die Sonomorphologie der Gichttophi ist schon lange bekannt und ähnelt der Morphologie der oben beschriebenen intraartikulären Harnsäurekonglomerate. Gichttophi erscheinen ebenfalls wolkig echoreich mit partieller oder kompletter dorsaler Schallauslöschung. Wenn sie viel Flüssigkeit enthalten und damit auch von der Konsistenz her weicher sind, können sie auch echoärmer sein.

Die Sonographie ermöglicht es zudem, Flüssigkeit in Gelenken, Bursen, Sehnenscheiden oder Tophi zu finden, die dann entweder sonographiegestützt oder sonographiegesteuert punktiert werden können, d. h. die Punktion erfolgt nach sonographischer Markierung bzw. unter sonographischer Sicht. Das Punktat wird dann unter dem $>$ Polarisationsmikroskop untersucht, um Harnsäurekristalle nachzuweisen, die pathognomonisch für die Erkrankung sind. Außerdem wird die Zellzahl im Punktat bestimmt, es erfolgt eine Zelldifferenzierung sowie eine Untersuchung auf bakterielle Erreger, da akute Gicht und eitrige Arthritis oder Weichteilinfektionen oft klinisch sehr ähnlich sind. Finden sich sonographisch intra- oder extraartikulär keine punktablen Flüssigkeitsansammlungen in Strukturen, in denen Harnsäurekonglomerate vermutet werden, kann die Region dennoch unter 
Abb. 6 Dorsaler Längsschnitt eines Kniegelenks. Im Knorpel am Epicondylus lateralis femoris finden sich echoreiche Strukturen $(\rightarrow)$, die Konglomerate von Pyrophosphatkristallen repräsentieren

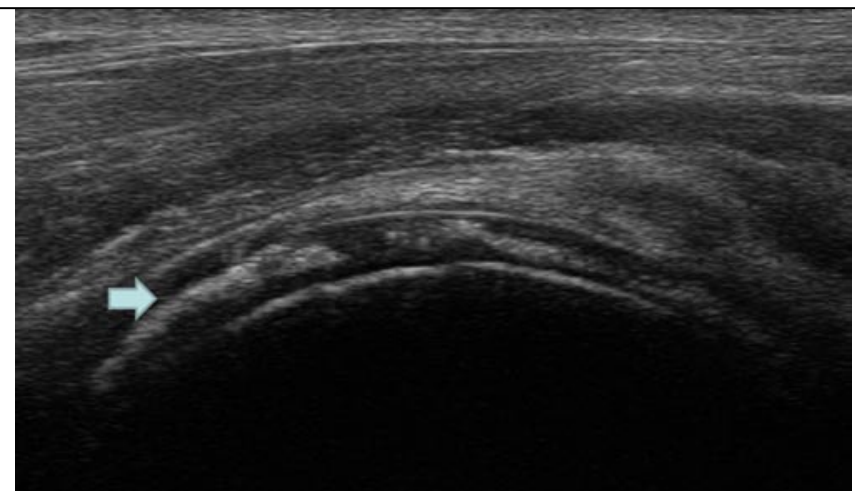

sonographischer Sicht punktiert werden. Zunächst werden je nach Region 1-4 ml physiologische Kochsalzlösung injiziert. Dann genügt die Aspiration eines Tropfens Spülflüssigkeit, um die Harnsäurekristalle polarisationsmikroskopisch nachweisen zu können.

Unter suffizienter harnsäuresenkender Therapie mit Zielwerten für den Harnsäurespiegel unter $360 \mathrm{mmol} / \mathrm{l}$ bzw. $6 \mathrm{mg} / \mathrm{dl}$ bilden sich die sonographischen Veränderungen einschließlich des Doppelkonturzeichens zurück bzw. verschwinden komplett $[26,40]$. Während sich Harnsäurekristalle extraartikulär, im Synovium oder auf dem Knorpel ablagern, finden sich Pyrophosphatkristalle bei Chondrokalzinose innerhalb des Knorpels. Sonographisch lassen sich die Ablagerungen als echoreiche Linien oder Punkte besonders im Knorpel des Kniegelenks (• Abb. 6), häufig auch am Humeruskopf und im triangulären Knorpel des Ulnokarpalgelenks darstellen. Die Veränderungen sind pathognomonisch für die Chondrokalzinose $[12,14]$. Der sonographische Nachweis von Pyrophosphatkristallen im Knorpel von Kniegelenken ist häufig mit dem Nachweis von Kniegelenkergüssen assoziiert [15].

MRT und Computertomographie (CT) sind alternativ anwendbare Schnittbildverfahren. Die Ergebnisse korrelieren gut mit den Sonographiebefunden. Es gibt bisher wenige Vergleichsstudien zur Beurteilung der Sensitivität und Spezifität dieser drei Verfahren für den Nachweis von gichttypischen Kristallansammlungen [11]. Mittels CT lassen sich diese vermutlich am sensitivsten darstellen, MRT und Sonographie dürften ähnlich sensitiv und spezifisch sein. Die Sonographie hat gegenüber CT und MRT folgende Vorteile:

- Der Rheumatologe kann sie selbst in seiner Praxis oder Klinik durchführen.

- Er kann den bildgebenden Befund zeitgleich mit der klinischen Untersuchung bewerten.

- Er kann in der gleichen Sitzung die gezielte Punktion durchführen, ggf. mit vorheriger Spülung der Region mit physiologischer Kochsalzlösung.

\section{Schlussfolgerung}

Die Sonographie bildet charakteristische Befunde bei der Gicht ab, wie wolkig echoreiche Strukturen mit partieller oder kompletter dorsaler Schallauslöschung und echoreiche Ablagerungen auf dem Knorpel. Bei der Chondrokalzinose finden sich echoreiche Strukturen dagegen innerhalb des Knorpels. Zusätzlich bietet die Sonographie die Möglichkeit zum Auffinden punktabler Regionen für die weitere Diagnostik. Sie hat damit heutzutage eine zentrale Stellung in der Diagnostik der Gicht durch den Rheumatologen erlangt.

\section{Interventionen}

Die Sonographie des Bewegungsapparates erleichtert mittels sonographiegesteuerter Interventionen therapeutische Maßnahmen. Zum einen kann eine Pathologie, wie z. B. eine Synovitis, eine Tenosynovitis oder eine Bursitis, genau lokalisiert werden. Zum anderen wird die Detektionsrate pathologischer Befunde und dadurch der Erfolg einer Gelenkpunktion zwecks Bestimmung der Zellzahl, zur Kristalldiagnostik und für mikrobiologische Untersuchungen signifikant erhöht [7]. Der nicht immer vorhandende klinische Erfolg einer nichtbildgebend gesteuerten („blinden“) Injektion ist erklärbar durch die sehr variable Trefferquote, je nach Vorerfahrung des Rheumatologen und injizierter Struktur. Ultraschallgesteuerte intraartikuläre und Weichteilinjektionen führten in klinischen Studien zu einer höheren Ansprechrate als „blinde“ Injektionen [33].
Bei Chondrokalzinose finden sich Pyrophosphatkristalle innerhalb des Knorpels

Die Sonographie hat eine zentrale Stellung in der Diagnostik der Gicht erlangt

Die Detektionsrate pathologischer Befunde wird durch den Ultraschall signifikant erhöht 

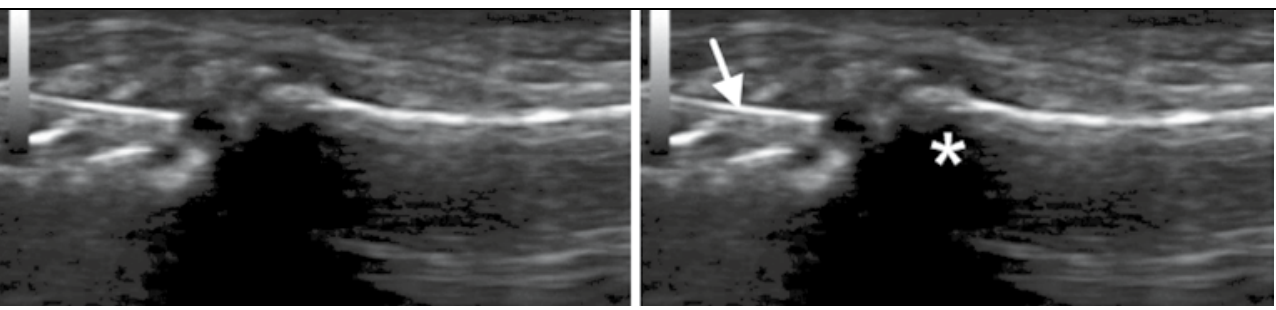

Abb. 7 Dorsolateraler Längsschnitt des PIP-Gelenks. Injektion in das PIP-Gelenk (Pfeil: Nadel, Stern: Basis der mittleren Phalanx)
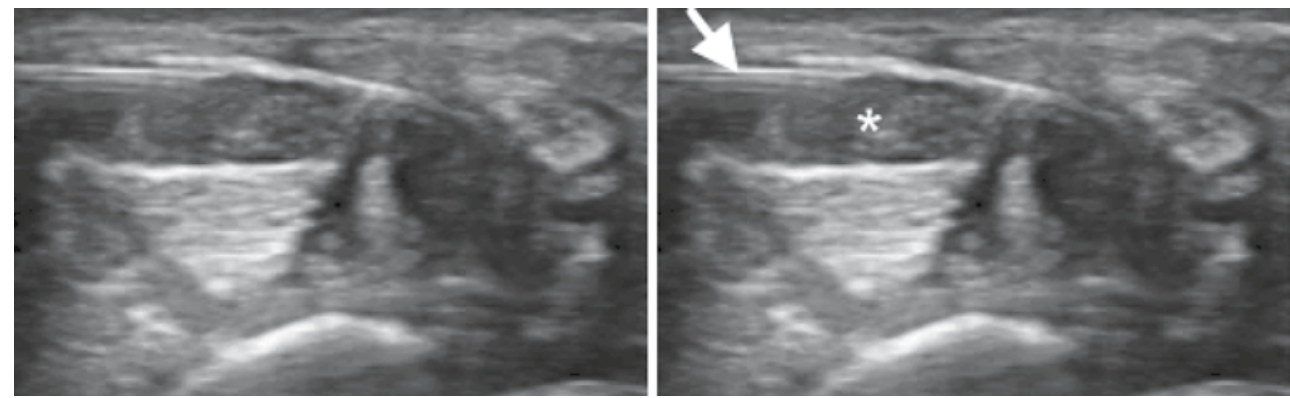

Abb. $8 \Delta$ Palmare Transversalschnittebene am Handgelenk. Injektion unterhalb des Retinaculum flexorum (Pfeil: Nadel, Stern: N. medianus)
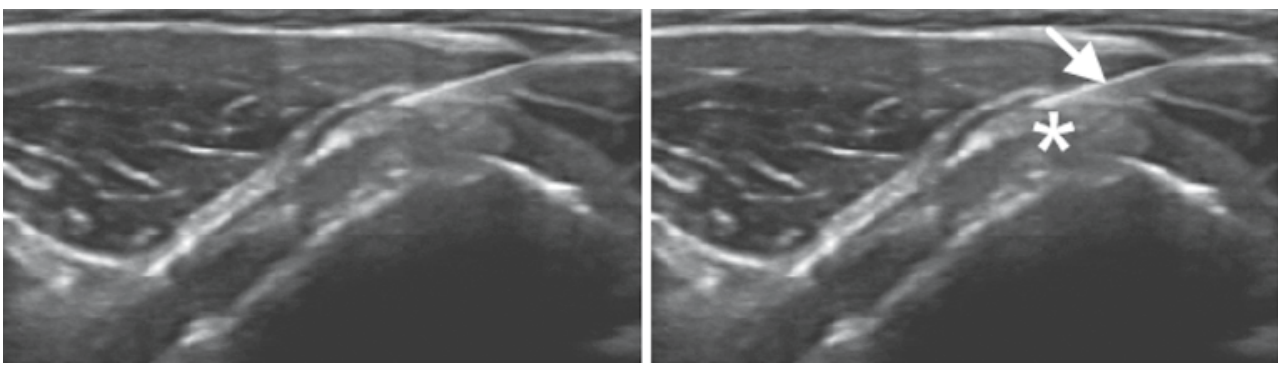

Abb. $9 \Delta$ Ventraler Transversalschnitt der Schulter, Arm in Außenrotation. Injektion in die Bursa bei ansatznaher Verkalkung (Stern) der Subscapularissehne (Pfeil: Nadel)

\section{- Gesteuerte Interventionen}

Durch eine präzise Platzierung der Nadel erhöht sich der Erfolg einer Gelenkpunktion signifikant

\section{- Ganglien \\ Karpaltunnelsyndrom}

Unter gesteuerten Interventionen verstehen wir die Markierung (z. B. mit einem Kugelschreiber oder einer Büroklammer) und Planung einer Punktionsstelle mittels Ultraschall oder gezielte Punktionen und Infiltrationen unter direkter sonographischer Sicht unter Einhaltung einer aseptischen Technik (z. B. sterile Schutzhülle über der Sonde oder steriles Einpacken des Schallkopfes unter Anwendung von sterilem Gel). Durch eine präzisere Platzierung der Nadel erhöht sich der Erfolg einer Gelenkpunktion im Vergleich zur klinisch gesteuerten Punktion signifikant: z. B. 25\% vs. 100\% für die Schulter, $40 \%$ vs. 95\% für das Knie und 20\% vs. 100\% für das Sprunggelenk [5]. Durch zusätzliche Injektion von Luft unter Sicht kann zudem die korrekte Lage der Nadel überprüft werden.

\section{Interventionen an der oberen Extremität}

Diagnostische Punktionen in den PIP-Gelenken (• Abb. 7) und in den MCP-Gelenken unter Ultraschallsicht (96\%) sind im Vergleich zur palpatorisch gesteuerten Punktion (59\%) erfolgreicher.

Bei Patienten mit aktiver RA wurde das proximale Handgelenk zwischen Os lunatum und Radius ultraschallgesteuert therapeutisch infiltriert bei gleichzeitiger Applikation von Gadolinium. Die im Anschluss veranlasste MRT des Handgelenks bestätigte die korrekte Applikation, wobei sich das Kontrastmittel je nach Synovitis-Score in der MRT unterschiedlich in den vier Kompartimenten des Handgelenks verteilte. An den Fingergelenken eignet sich der Ultraschall zum Monitoring therapeutischer Maßnahmen, wie beispielsweise zum Nachweis des Rückgangs von Synovitiden nach ultraschallgesteuerter Injektion bei RA. Des Weiteren können an der Hand \ Ganglien gezielt infiltriert werden, beim - Karpaltunnelsyndrom können sowohl Ursachen des „Entrapments“ dargestellt als auch gezielte Injektionen unterhalb des Retinaculums (• Abb. 8) sicher durchgeführt werden. 
Ultraschallgesteuerte Interventionen an der Schulter (• Abb. 9) führen im Vergleich zu blind durchgeführten Injektionen zu einem besseren Ansprechen gemessen am Schmerz oder an der Verbesserung der Beweglichkeit [8]. Bei „blinden“ Schulterinjektionen wurde in einer Arbeit von Naredo et al. [25] festgestellt, dass nur knapp ein Drittel der subakromial und nur knapp 40\% der glenohumeral vorgesehenen Injektionen korrekt appliziert wurden. In einer anderen Arbeit von Jones et al. [18] waren nur ein Viertel der Injektionen am gewünschten Ort. Dies spiegelte sich in einem signifikanten Unterschied im klinischen Ansprechen wider. Die Injektion unter direkter Sicht über einen anterolateralen Zugangsweg eignet sich zudem für die Kontrastmittelinjektion zur $>$ MRTArthrographie der Schulter.

Mehrere Fallserien haben eine gute Erfolgsrate der ultraschallgezielten Perforation und Spülung bei kalzifizierenden Tendinosen an der Schulter zeigen können. Die Behandlung von Verkalkungen der Rotatorenmanschette unter sonographischer Sicht ist eine sichere und erfolgreiche Methode in der Hand eines erfahrenen interventionellen Rheumatologen. Das „Needling“ ist nicht nur eine effektive und sichere Therapiemethode bei Schulterkalzifikationen, sondern kann auch in anderen Körpersehnen erfolgreich eingesetzt werden.

\section{Interventionen an der unteren Extremität}

Bei Patienten mit Hüftprothesen und vermuteter septischer Koxitis zeichnet sich die ultraschallgesteuerte Punktion durch eine hohe Erfolgrate der diagnostischen Arthrozentese aus. Auch bei aktivierter Koxarthrose kann eine ultraschallgesteuerte Injektion zu einer signifikanten Schmerzlinderung führen. Eine erfolgreiche Testinfiltration ins Hüftgelenk mit Lokalanästhetika klärt in vielen unklaren Fällen die eigentliche Schmerzquelle. Im Kniegelenk sind Ergussmengen ab 7 ml sonographisch erfassbar, was sensitiver ist als die klinische Untersuchung: Klinisch werden etwa 30\% der Kniesynovitiden nicht erfasst.

Ultraschallgesteuerte Injektionen an Fußgelenken zeigten in einer Kadaverstudie sehr hohe Trefferquoten. In den MTP-Gelenken ist die sonographische Detektionsrate von Gelenkergüssen bis zu 10-mal höher als in der klinischen Untersuchung [42]. Weitere erfolgreiche und technisch einfach und schnell durchführbare Injektionen an der unteren Extremität sind die Behandlung einer $>$ Bursitis subachillea, einer $>$ Plantarfasziitis oder eines Morton-Pseudoneurinoms.

\section{Interventionen an der Wirbelsäule und im Beckenbereich}

Im klinischen Alltag hat sich die ultraschallgesteuerte Injektion des M. piriformis und des SIG als sichere und einfache Methode etabliert. Aufgrund der fehlenden Strahlenbelastung erlaubt diese Methode auch, schwangere Patientinnen zu behandeln. In einer Arbeit, in der 60 SIG im synovialen Anteil unter Ultraschallsicht therapeutisch infiltriert wurden, lagen mittels Bildverstärkerkontrolle 76,6\% intraartikulär. Das Ansprechen auf die Intervention bei einer Arthritis des SIG nahm im Verlauf von $60 \%$ auf $93,5 \%$ zu. Demgegenüber liegen nur 40\% der im mittleren bis oberen Drittel durchgeführten SIG-Injektion gemessen an der MRT-Kontrolle intraartikulär. Die Ansprechrate wurde davon nicht beeinflusst.

\section{Fazit für die Praxis}

Es können folgende Vorteile der sonographiegesteuerten Punktion und Injektion gegenüber dem klinisch gesteuerten Vorgehen festgestellt werden:

- exaktere Differenzierung und höhere Detektionsrate pathologischer Befunde wie z. B. von Synovitiden, Tenosynovitiden oder Bursitiden,

- genauere Lokalisation einer Pathologie und Platzierung der Nadel und somit erfolgreichere diagnostische Gelenkpunktionen,

- erhöhtes Ansprechen auf therapeutische Injektionen.
Ultraschallgesteuerte Interventionen führen zu einem besseren klinischen Ansprechen

\section{MRT-Arthrographie}

Die Behandlung von Verkalkungen der Rotatorenmanschette unter sonographischer Sicht ist eine sichere und erfolgreiche Methode

\section{- Septische Koxitis}

Im Kniegelenk sind Ergussmengen ab $7 \mathrm{ml}$ sonographisch erfassbar

\section{- Bursitis subachillea \\ - Plantarfasziitis}

Die ultraschallgesteuerte Injektion des M. piriformis und des SIG hat sich als sichere und einfache Methode etabliert 


\section{Korrespondenzadresse}

\section{Dr. G. Tamborrini}

Rheumaklinik, UniversitätsSpital Zürich

Gloriastr. 25, 8091 Zürich

giorgio.tamborrini@sec.usz.ch

Interessenkonflikt. Die Autorinnen und Autoren geben an, dass kein Interessenkonflikt besteht.

\section{Literatur}

1. Arslan $\mathrm{H}$, Sakarya ME, Adak B et al (1999) Duplex and color Doppler sonographic findings in active sacroiliitis. AJR Am J Roentgenol 173:677680

2. Backhaus M, Burmester GR, Sandrock D et al (2002) Prospective twoyear follow-up study comparing novel and conventional imaging procedures in patients with arthritic finger joints. Ann Rheum Dis 61:895-904

3. Backhaus M, Kamradt T, Sandrock D et al (1999) Arthritis of the finger joints: a comprehensive approach comparing conventional radiography, scintigraphy, ultrasound, and contrast-enhanced magnetic resonance imaging. Arthritis Rheum 42:1232-1245

4. Backhaus M, Schmidt WA, Mellerowicz H et al (2002) Technik und Stellenwert in der Arthrosonographie in der rheumatologischen Diagnostik - Teil 6: Sonographie der Hand- und Fingergelenke. Z Rheumatol 61:674687

5. Balint PV, Kane D, Hunter J et al (2002) Ultrasound guided versus conventional joint and soft tissue fluid aspiration in rheumatology practice: a pilot study. J Rheumatol 29:2209-2213

6. Balint PV, Kane D, Wilson $\mathrm{H}$ et al (2002) Ultrasonography of entheseal insertions in the lower limb in spondyloarthropathy. Ann Rheum Dis 61:905-910

7. Bruyn GAW, Schmidt WA (2009) How to perform ultrasound-guided injections. Best Pract Res Clin Rheumatol 23: $269-279$

8. Chen MJ, Lew HL, Hsu TC et al (2006) Ultrasound-guided shoulder injections in the treatment of subacromial bursitis. Am J Phys Med Rehabil 85:31-35

9. D'Agostino MA, Aegerter $P$, JousseJoulin S et al (2009) How to evaluate and improve the reliability of power Doppler ultrasonography for assessing enthesitis in spondylarthritis. Arthritis Rheum 61:61-69

10. D'Agostino MA, Breban M, SaidNahal R, Dougados M et al (2002) Refractory inflammatory heel pain in spondylarthropathy: a significant response to infliximab documented by ultrasound. Arthritis Rheum 46:840841, author reply 841-843
11. Dalbeth N, McQueen FM (2009) Use of imaging to evaluate gout and other crystal deposition disorders. Curr Opin Rheumatol 21:124-131

12. Dufauret-Lombard $C$, Vergne-Salle $P$, Simon A et al (2010) Ultrasonography in chondrocalcinosis. Joint Bone Spine 77:218-221

13. Filippucci E, Aydin SZ, Karadag O et al (2009) Reliability of high-resolution ultrasonography in the assessment of Achilles tendon enthesopathy in seronegative spondyloarthropathies. Ann Rheum Dis 68:18501855

14. Filippucci E, Riveros MG, Georgescu D et al (2009) Hyaline cartilage involvement in patients with gout and calcium pyrophosphate deposition disease. An ultrasound study. Osteoarthritis Cartilage 17:178-181

15. Filippucci $E$, Scirè $C A$, Delle Sedie $A$ et al (2010) Ultrasound imaging for the rheumatologist. XXV. Sonographic assessment of the knee in patients with gout and calcium pyrophosphate deposition disease. Clin Exp Rheumatol 28:2-5

16. Fiocco U, Ferro F, Vezzù M et al (2005) Rheumatoid and psoriatic knee synovitis: clinical, grey scale, and power Doppler ultrasound assessment of the response to etanercept. Ann Rheum Dis 64:899-905

17. Fournié B, Margarit-Coll N, Champetier de Ribes TL et al (2006) Extrasynovial ultrasound abnormalities in the psoriatic finger. Prospective comparative power-doppler study versus rheumatoid arthritis. Joint Bone Spine 73:527-531

18. Jones A, Regan M, Ledingham J et al (1993) Importance of placement of intra-articular steroid injections. $\mathrm{Br}$ Med J 307:1329-1330

19. Karim Z, Wakefield RJ, Conaghan PG et al (2001) The impact of ultrasonography on diagnosis and management of patients with musculoskeletal conditions. Arthritis Rheum 44:2932-2933

20. Klauser A, Halpern EJ, Frauscher $F$ et al (2005) Inflammatory low back pain: high negative predictive value of contrast-enhanced color Doppler ultrasound in the detection of inflamed sacroiliac joints. Arthritis Rheum 53:440-444
21. Lehtinen A, Taavitsainen M, Leirisalo-Repo M (1994) Sonographic analysis of enthesopathy in the lower extremities of patients with spondylarthropathy. Clin Exp Rheumatol 12:143-148

22. McGonagle D, Gibbon W, Emery P (1998) Classification of inflammatory arthritis by enthesitis. Lancet 352:1137-1140

23. McGonagle D, Marzo-Ortega $\mathrm{H}$, O'Connor P et al (2002) The role of biomechanical factors and HLA-B27 in magnetic resonance imaging-determined bone changes in plantar fascia enthesopathy. Arthritis Rheum 46(2):489-493

24. McGonagle D, Wakefield RJ, Tan AL et al (2008) Distinct topography of erosion and new bone formation in achilles tendon enthesitis: implications for understanding the link between inflammation and bone formation in spondylarthritis. Arthritis Rheum 58:2694-2699

25. Naredo E, Cabero F, Beneyto P et al (2004) A randomized comparative study of short term response to blind injection versus sonographic-guided injection of local corticosteroids in patients with painful shoulder. J Rheumatol 31:308-314

26. Perez-Ruiz F, Martin I, Canteli B (2007) Ultrasonographic measurement of tophi as an outcome measure for chronic gout. J Rheumatol 34:1888-1893

27. Rettenbacher T, Ennemoser S, Weirich $\mathrm{H}$ et al (2008) Diagnostic imaging of gout: comparison of high-resolution US versus conventional $X$ ray. Eur Radiol 18:621-630

28. Ribbens C, Andre B, Marcelis S et al (2003) Rheumatoid hand joint synovitis: gray-scale and power Doppler US quantifications following anti-tumor necrosis factor-alpha treatment: pilot study. Radiology 229:562-569

29. Scheel AK, Hermann KG, Kahler E et al (2005) A novel ultrasonographic synovitis scoring system suitable for analyzing finger joint inflammation in rheumatoid arthritis. Arthritis Rheum 52:681-686 
30. Scheel AK, Hermann KG, Ohrndorf Set al (2006) Prospective 7 year follow-up imaging study comparing radiography, ultrasonography and magnetic resonance imaging in rheumatoid arthritis finger joints. Ann Rheum Dis 65:595-600

31. Schell AK, Backhaus M (2004) Ultrasonographic assessment of the finger and toe joint inflammation in rheumatoid arthritis: comment on the article by Szkudlarek et al [letter]. Arthritis Rheum 50:1008

32. Schlesinger $\mathrm{N}$, Thiele RG (2010) The pathogenesis of bone erosions in gouty arthritis. Ann Rheum Dis 69:1907-1912

33. Sibbitt WL, Speisajovich A, Michael AA et al (2009) Does sonographic needle guidance affect the clinical outcome of intraarticular injections? J Rheumatol 36:9

34. Strunk J (2006) Farbdoppler - Sonographie der Gelenke. Akt Rheumatol 31:148-156

35. Szkudlarek M, Court-Payen M, Jacobsen $S$ et al (2003) Interobserver agreement in ultrasonography of the finger and toe joints in rheumatoid arthritis. Arthritis Rheum 48:955-962

36. Szkudlarek M, Narvestad E, Klarlund $M$ et al (2004) Ultrasonography of the metatarsophalangeal joints in rheumatoid arthritis: comparison with magnetic resonance imaging, conventional radiography, and clinical examination. Arthritis Rheum 50:2103-2112
37. Szkudlarek MKM, Narvestad E, CourtPayen M et al (2004) Ultrasonography of the ra finger joints is more sensitive than conventional radiography for detection of erosions without loss of specificity, with MRI as a reference method. Ann Rheum Dis 63 (Suppl 1):82-83

38. Terslev L, Torp-Pedersen S, Savnik A et al (2003) Doppler ultrasound and magnetic resonance imaging of synovial inflammation of the hand in rheumatoid arthritis: a comparative study. Arthritis Rheum 48:24342441

39. Thiele RG, Schlesinger N (2007) Diagnosis of gout by ultrasound. Rheumatology 46:1116-1121

40. Thiele RG, Schlesinger N (2010) UItrasonography shows disappearance of monosodium urate crystal deposition on hyaline cartilage after sustained normouricemia is achieved. Rheumatol Int 30:495-503

41. Unlu E, Pamuk ON, Cakir N (2007) Color and duplex Doppler sonography to detect sacroiliitis and spinal inflammation in ankylosing spondylitis. Can this method reveal response to anti-tumor necrosis factor therapy? J Rheumatol 34:110-116

42. Wakefield RJ, Karim Z, Sonaghan PG et al (1999) Sonography is more sensitive at detecting synovitis in the metatarsophalangeal joints than clinical examination. Arthritis Rheum 42(Suppl 9):1688
43. Wakefield RJ, Balint P, Szkudlarek M et al (2005) Proceedings form the OMERACT special interest group for musculoskeletal ultrasound including definitions for ultrasonographic pathology. J Rheumatol 32:24852487

44. Wakefield RJ, Gibbon WW, Conaghan PG et al (2000) The value of sonography in the detection of bone erosions in patients with rheumatoid arthritis. Arthritis Rheum 43:27622770

45. Walther M, Harms H, Krenn V et al (2001) Correlation of power Doppler sonography with vascularity of the synovial tissue of the knee joint in patients with osteoarthritis and rheumatoid arthritis. Arthritis Rheum 44:331-338

46. Wright SA, Filippucci E, McVeigh C et al (2007) High-resolution ultrasonography of the first metatarsal phalangeal joint in gout: a controlled study. Ann Rheum Dis 66:859-864 


\section{CME-Fragebogen}

\section{kostenfreie Teilnahme für Abonnenten}

Bitte beachten Sie:

- Antwortmöglichkeit nur online unter: CME.springer.de

- Die Frage-Antwort-Kombinationen werden online individuell zusammengestellt.

- Es ist immer nur eine Antwort möglich.

\section{Eine 23-jährige Frau leidet seit 6 Monaten an Beschwerden in beiden Handgelenken insbe- sondere in den frühen Morgen- stunden. Die Röntgenaufnah- men beider Hände und Vorfü- Be sind unauffällig. Klinisch zeigt sich eine Schwellung im ulnaren Handgelenksbereich. Was verbirgt sich sonogra- phisch am ehesten dahinter? \\ $\square$ Eine Bursitis ulnae. \\ $\square$ Eine Tenosynovitis der Exten- sor-carpi-ulnaris-Sehne. \\ $\square$ Eine Erosion am Processus styloideus. \\ $\square$ Eine Karpalsynovialitis. \\ $\square$ Ein Ganglion.}

Eine 31-jährige Frau mit bekannter RA kommt mit einem geschwollenen Kniegelenk in die Rheumaambulanz. Welchen sonomorphologischen Befund erwarten Sie am ehesten?

$\square$ Eine Ruptur der Quadrizepssehne.

$\square$ Eine Enthesitis der Patellarsehne.

$\square$ Eine Synovialitis mit Erguss und synovialer Kapselverdickung im oberen Recessus.

$\square$ Erosionen in der präpatellaren Gleitrinne.

$\square$ Eine subkutane ödematöse Gewebeschwellung.

Eine 63-jährige Frau mit RA klagt über Schulterschmerzen. Der Schulter-Nacken-Griff ist nur erschwert möglich. Welcher Befund im Ultraschall passt am besten?
Tenosynovialitis der langen Bizepssehne.

$\square$ Verdickte Bursa subdeltoidea.

Erosionen am Humeruskopf.

$\square$ Teilruptur der Supraspinatussehne.

Feine Verkalkung am Ansatz der Supraspinatussehne.

Ein 25-jähriger Mann leidet seit einem Jahr an nächtlichen tieflumbalen Schmerzen. Er ist HLA-B27-positiv, die Röntgenaufnahme zeigt entzündliche Veränderungen der Sakroiliakalgelenke. Wie groß ist die Wahrscheinlichkeit, dass mittels hochauflösendem Ultraschall (B-Bild und Doppler) pathologische Enthesen an den unteren Extremitäten gefunden werden?
$\square$ 60-70\%
ㅁ $20-30 \%$
$\square$ 90-100\%
口 $40-50 \%$
$\square$ 50-60\%

Eine 35-jährige Frau mit bekannter kutaner Psoriasis meldet sich mit einem wurstförmig aufgetriebenen Mittelfinger rechts. Welchen sonomorphologischen Befund erwarten Sie am ehesten?

$\square$ Synovitiden von MCP- und PIPGelenken.

$\square$ Eine Enthesitis.

$\square$ Einen periostalen Knochenanbau mit Mehrdurchblutung.

Eine Tenosynovitis der Beugesehnen.

Eine subkutane Gewebevermehrung.
Ein 45-jähriger Marathonläufer meldet sich wegen Schmerzen in der rechten Achillessehne. Die Sehne ist etwa $2 \mathrm{~cm}$ proximal des Fersenbeins verdickt. Welcher Befund im Ultraschall passt am besten?

$\square$ Olivenförmige Auftreibung der Achillessehne im Sehnenkörper, Vaskularisierung in und neben der Sehne vermehrt.

$\square$ Verdickte Bursa praeachillea mit Mehrdurchblutung.

$\square$ Sehnenansatz mit hypoechogener Zone und Mehrdurchblutung.

$\square$ Erosion am Fersenbein proximal des Achillessehnenansatzes.

$\square$ Feine Verkalkung am Achillessehnenansatz.

Welcher sonographische Befund ist nicht typisch für eine Gicht?

$\square$ Echoreiche Synovialisverbreiterung.

$\square$ Doppelkonturzeichen.

$\square$ Echoreiche Linien im Knorpel.

$\square$ Partielle dorsale Schallauslöschung von intraartikulären Strukturen.

$\square$ Echoreiche Punkte im Knorpel.

Welcher Befund ist typisch für eine Chondrokalzinose?

$\square$ Bizarre Erosionen am Humeruskopf.

$\square$ Echoreiche Punkte im Knorpel des Kniegelenks.

Sichelförmige echoreiche Strukturen in der Rotatorenmanschette.
Osteophyten am Kniegelenkspalt.

$\square$ Kombination von Usuren und Osteophyten.

Wie viele der blind durchgeführten subakromialen Injektionen werden korrekt in die Bursa appliziert?
- $10-20 \%$
$25-33 \%$
口 $50-80 \%$
$\square 95 \%$
$100 \%$

Welches ist kein Vorteil der sonographiegesteuerten Punktion oder Injektion?

$\square$ Erhöhtes Ansprechen auf therapeutische Injektionen.

$\square$ Genauere Lokalisation einer Pathologie und Platzierung der Nadel und somit erfolgreichere diagnostische Gelenkpunktionen.

Weniger Komplikationen (z. B. Infektion) gegenüber nichtbildgebenden Techniken.

Durch zusätzliche Injektion von Luft unter Sicht kann die korrekte Lage der Nadel überprüft werden.

$\square$ Es besteht die Möglichkeit einer gezielten Biopsie.

Diese Fortbildungseinheit ist

12 Monate auf

CME.springer.de verfügbar.

Den genauen Einsendeschluss

erfahren Sie unter

CME.springer.de 